\title{
Multi-vehicle Control and Optimization for Spatiotemporal Sampling
}

\author{
Nitin Sydney and Derek A. Paley
}

\begin{abstract}
In this paper we analyze the mapping accuracy of a sensor network using a quantitative measure of the mapping error as performance metric. We use optimal interpolation to calculate the estimation error of a map of a spatiotemporal field produced by assimilating observations collected by a group of vehicles. The vehicles travel in a closed trajectory in a steady, uniform flowfield. The mapping error is analyzed for statistically homogeneous fields and for inhomogeneous fields in which the correlation coefficient depends on position. For the homogeneous field, we design a closed-loop speed controller to minimize the average mapping error and, for the inhomogeneous field, we introduce an artificial flowfield to minimize a convex combination of the average error and maximum error.
\end{abstract}

\section{INTRODUCTION}

In the fields of oceanography and meteorology it is critical to have accurate maps of a variety of quantities such as salinity, temperature, and wind speed. However, the available sensing resources might not be adequate to accurately map the quantity using a static sensor distribution. For example, consider the task of measuring wind speed in an impending severe-weather front. Weather systems can change significantly in a short period of time, so the deployment window and the available time to collect measurements may be short [1]. In addition, certain areas of the windfield may vary significantly and require more attention. For this type of system, we require a mobile sensor network with the ability to map critical areas accurately.

We study the use of a multi-vehicle network to optimally map a scalar spatiotemporal field with respect to a quantitative error metric. The scalar field is treated as a random field with known statistics. In related work [2], the authors consider a persistent monitoring task in which a changing environment is modeled using a general accumulation function. In [3], the error metric is related to the mapping performance and vehicles are routed to maximize the mapping skill. This metric applies a framework known as objective analysis. Another approach is to assume that the field follows a known process model, such as diffusion, and to estimate the model parameters. [4], [5], and [6] provide algorithms to perform distributed parameter estimation. Optimal placement of a static sensor network to cover a time-invariant field has been studied in [7].

This material is based upon work supported by the National Science Foundation under Grant No. CMMI0928416 and the Office of Naval Research under Grant No. N00014-09-1-1058.

Nitin Sydney is a graduate student in the Department of Aerospace Engineering at the University of Maryland, College Park, MD, 20742 nsydney@umd. edu

Derek A. Paley is an Assistant Professor in the Department of Aerospace Engineering at the University of Maryland, College Park, MD, 20742 dpaley@umd.edu
Schemes to compute the mapping error of a scalar field typically rely on a priori information. In [3], the first-order and second-order statistics of the field are needed and, in [4, $6,5]$, parameter estimation requires a process model for the spatiotemporal field. Determining the statistics of the field may conflict with minimizing the mapping error. There is also the need to analyze the mapping error if the statistics of the field can vary in time and space, i.e., the field is inhomogeneous.

In this paper we use optimal interpolation to perform mapping-error analysis on spatiotemporal fields with known first-order and second-order statistics. The sensor platforms travel on a periodic trajectory using a steering control law [8] to remain evenly spaced in time (a time-splay formation) in a steady, uniform flowfield. We use metrics corresponding to the maximum and average error to determine the mapping performance of a sensor network in a homogeneous field. A closed-loop vehicle speed controller is derived to minimize the average mapping error in a uniform flow. We then optimize the performance of the sensor network in an inhomogeneous field for which the correlation coefficient depends on position. An artificial flowfield is introduced to minimize a convex combination of the maximum and average error.

The contributions of this paper are 1) a framework based on optimal interpolation theory to evaluate sampling performance in homogeneous and inhomogeneous spatiotemporal fields; 2) a closed-loop speed controller that minimizes the average mapping error in a homogeneous field in the presence of a uniform flowfield; and 3) an open-loop speed controller based on an artificial flowfield that minimizes the maximum and/or average mapping error in an inhomogeneous field.

Section II summarizes relevant background information, including an overview of mapping-error analysis and sensor dynamics and control. Section III provides mapping-error analysis for closed trajectories in a spatiotemporal field. Section IV provides speed control laws that optimize the sampling performance by minimizing the mapping error. Section V summarizes the paper and ongoing work.

\section{BACKGROUND}

\section{A. Quantification of Mapping Error}

We use mapping error to quantify the performance of a sensor network. To analyze the mapping error, we use an optimal interpolation (OI) framework, formerly known as objective analysis, developed in [9] and popularized in [10]. OI treats the value of a scalar field at a point, $A(\mathbf{x}) \triangleq A_{\mathbf{x}} \in$ $\mathbb{R}$, where $\mathbf{x} \in \mathbb{R}^{n}(n \in \mathbb{N})$, as a random variable. We would 
like to estimate the value of the field at a point $\mathrm{x}$ using noisy observations of $A(\mathbf{x})$. The components of $\mathbf{x}$ are spacetime coordinates. For example, in a one-dimensional space, $\mathbf{x}=\left[\begin{array}{ll}x & t\end{array}\right]^{T}$, where $x$ is position and $t$ is time.

In order to produce the optimal estimate, $\hat{A}_{\mathbf{x}}$, we require the mean of the field, $E\left[A_{\mathbf{x}}\right] \triangleq \bar{A}_{\mathbf{x}}$, and the covariance around the mean $E\left[\left(A_{\mathbf{x}}-\bar{A}_{\mathbf{x}}\right)\left(A_{\mathbf{x}^{\prime}}-\bar{A}_{\mathbf{x}^{\prime}}\right)\right] \triangleq C\left(\mathbf{x}, \mathbf{x}^{\prime}\right)$, where $E[\cdot]$ is the expected-value operator.

The field is estimated from $N$ noisy measurements, $z_{m}$, of the form

$$
z_{m}=A_{\mathbf{x}}+\varepsilon_{m}, \quad m=1, \ldots, N,
$$

where $\varepsilon_{m}$ is the measurement error. We assume $E\left[\varepsilon_{m} z_{l}\right]=0$ for all pairs $m$ and $l$ and $E\left[\varepsilon_{m} \varepsilon_{l}\right]=\tilde{\sigma}_{0} \delta_{m l} . \tilde{\sigma}_{0}$ is the standard deviation of the corrupting noise and $\delta_{m l}$ is the Kronecker delta eaual to 1 if $m=l$ and 0 otherwise.

From the Gauss-Markov theorem [11], the optimal estimate of the field is

$$
\hat{A}_{\mathbf{x}}=\bar{A}_{\mathbf{x}}+\sum_{m=1}^{N} C\left(\mathbf{x}, \mathbf{x}_{m}\right)\left(\sum_{l=1}^{N} M_{m l}^{-1} z_{m}\right) .
$$

$M_{m l}^{-1}$ is the $(m, l)$ entry of the inverse of the measurement covariance,

$$
M_{m l}=E\left[z_{m} z_{l}\right]=C\left(\mathbf{x}_{m}, \mathbf{x}_{l}\right)+\tilde{\sigma}_{0} \delta_{m l} .
$$

$C\left(\mathbf{x}_{m}, \mathbf{x}_{l}\right)$ is a covariance function that describes the statistics of the field, discussed further in Section III. The variance of the error, called the mapping error [12], is

$$
C_{e}(\mathbf{x}, \mathbf{x})=C(\mathbf{x}, \mathbf{x})-\sum_{m=1}^{N} \sum_{l=1}^{N} C\left(\mathbf{x}, \mathbf{x}_{m}\right) M_{m l}^{-1} C\left(\mathbf{x}, \mathbf{x}_{l}\right) .
$$

(Derivations for equations (1) and (2) can be found in [10] and [13].)

Using the mapping error, we adopt the following metric to quantify the measurement performance [12] :

$$
\mathcal{E}_{\mathrm{av}}=\frac{\int_{D} C_{e}(\mathbf{x}, \mathbf{x}) d \mathbf{x}}{\int_{D} d \mathbf{x}},
$$

which is a measure of the average error in the domain $D$. Similarly, we can define a metric for the maximum error

$$
\mathcal{E}_{\max }=\max _{\mathbf{x} \in D}\left[C_{e}(\mathbf{x}, \mathbf{x})\right] .
$$

\section{B. Sensor Platform Dynamics and Control}

When analyzing the mapping error in a uniform flowfield, we assume that the vehicles are modeled as Newtonian particles (see [14], [8]). The equations of motion are [8]

$$
\begin{aligned}
& \dot{r}_{k}=v_{0} e^{i \theta_{k}}+\beta \\
& \dot{\theta}_{k}=u_{k}, \quad k=1, \ldots, N,
\end{aligned}
$$

where $r_{k}$ is the position of the $k^{t h}$ particle represented in complex coordinates, $\beta$ is the velocity of a uniform flowfield (aligned with the real axis without loss of generality), $\theta_{k}$ is the orientation of the $k^{t h}$ vehicle's velocity relative to the flow, $v_{0}$ is the vehicle speed relative to the flow and $u_{k}$ is a steering control input.
This system can also be represented in inertial coordinates as

$$
\begin{aligned}
& \dot{r}_{k}=s_{k} e^{i \gamma_{k}} \\
& \dot{\gamma}_{k}=\nu_{k},
\end{aligned}
$$

where $s_{k}=\left|e^{i \theta_{k}}+\beta\right|$ and $\gamma_{k}=\arg \left(e^{i \theta_{k}}+\beta\right) . \nu_{k}$ is the steering control input relative to the inertial frame.

If we drive particle $k$ in the absence of flow with a constant input $u_{k}=\omega_{0} v_{0}$, the particle will travel in a circle of radius $\left|w_{0}\right|^{-1}$. In the presence of a flowfield, circular motion occurs when $\dot{\gamma}_{k}=\nu_{k}=\omega_{0} s_{k}$, which can be integrated by separation of variables to obtain

$$
t=\frac{1}{\omega_{0}} \int_{0}^{\gamma_{k}} \frac{d \gamma}{s(\gamma)} .
$$

A time-phase variable $\psi_{k}$ denotes the progress of the $k^{t h}$ vehicle around a circular trajectory [8]:

$$
\psi_{k}=\frac{2 \pi}{\omega_{0} T} \int_{0}^{\gamma_{k}} \frac{d \gamma}{s(\gamma)},
$$

where $T$ is the period of a single revolution. Decentralized steering-only control laws exist to stabilize time-splay circular formations in which the vehicles are uniformly spaced in time [8].

Using speed control allows vehicles to control their spatial separation in a flowfield. In the following particle model each vehicle has the ability to control its speed as well as its turning rate. Taking the inertial derivative of $\dot{r}_{k}=s_{k} e^{i \gamma_{k}}$ yields

$$
\ddot{r}_{k}=\dot{s}_{k} e^{i \gamma_{k}}+s_{k} i e^{i \gamma_{k}} \dot{\gamma}_{k} .
$$

The force on the $k_{t h}$ particle can be represented as

$$
F_{k}=\xi_{k} e^{i \gamma_{k}}+\nu_{k} s_{k} i e^{\gamma_{k}},
$$

where $\xi_{k}$ and $\nu_{k}$ are control inputs corresponding to the inertial speed and steering controls respectively. Using Newton's second law (and assuming unit mass), (5) and (6) can be combined to give

$$
\dot{s}_{k} e^{i \gamma_{k}}+s_{k} i e^{i \gamma_{k}} \dot{\gamma}_{k}=\xi_{k} e^{i \gamma_{k}}+\nu_{k} s_{k} i e^{\gamma_{k}} .
$$

Equating the real and imaginary parts yields the equations of motion

$$
\begin{aligned}
\dot{r}_{k} & =s_{k} e^{i \gamma_{k}} \\
\dot{s}_{k} & =\xi_{k} \\
\dot{\gamma}_{k} & =\nu_{k} .
\end{aligned}
$$

Steering and speed control laws are derived for (7). However, in order to implement the control onboard a vehicle, the control laws must be transformed for use in the following dynamics:

$$
\begin{aligned}
& \dot{r}_{k}=v_{k} e^{i \theta_{k}}+\beta \\
& \dot{v}_{k}=T_{k} \\
& \dot{\theta}_{k}=\frac{R_{k}}{v_{k}}=u_{k}
\end{aligned}
$$




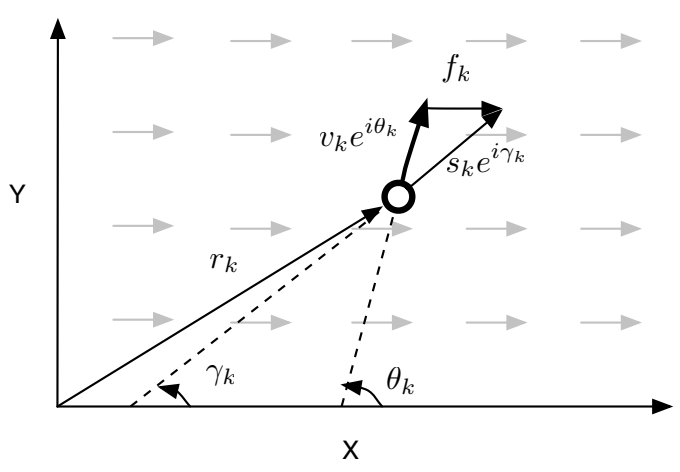

Fig. 1: Particle model in a uniform flowfield.

where $T_{k}$ is the speed control and $R_{k}$ is the steering control relative to the flow.

Using the geometry of the system shown in Figure 1 and the particle models given by (7) and (8), the transformation to express $T_{k}=T\left(\xi_{k}, \nu_{k}\right)$ and $R_{k}=R\left(\xi_{k}, \nu_{k}\right)$ is

$$
\begin{aligned}
R_{k} & =\frac{\nu_{k} s_{k}-\frac{s_{k} \xi_{k} \sin \left(\theta_{k}-\gamma_{k}\right)}{v_{k}+\beta \cos \theta_{k}}}{\cos \left(\theta_{k}-\gamma_{k}\right)+\frac{\beta \sin \theta_{k} \sin \left(\theta_{k}-\gamma_{k}\right)}{v_{k}+\beta \cos \theta_{k}}} \\
T_{k} & =\frac{s_{k} \xi_{k}+\beta \sin \theta_{k} R_{k}}{v_{k}+\beta \cos \theta_{k}} .
\end{aligned}
$$

With (9) and (10) any control derived in inertial coordinates can be transformed for implementation onboard a vehicle.

\section{ANALysis of MAPPING ERRoR}

\section{A. Homogeneous Field}

In this section, we analyze the mapping performance of vehicles traveling in a circular time-splay formation using the metrics defined in Section II-A. The performance is evaluated in a statistically homogeneous field characterized by the spatial and temporal decorrelation scales. We are interested in determining the mapping error along the path of vehicles. Vehicle trajectories are generated by (8) and finely discretized to be used in the generation of error maps using (2). The discretization spacing is chosen such that the mapping error calculation approaches the continuous path limit.

As discussed in Section II-A, a covariance function is needed a priori. We assume an exponential covariance function of the form [15]

$$
C\left(\mathbf{x}, \mathbf{x}^{\prime}\right)=\sigma_{0} e^{\frac{\left|x-x^{\prime}\right|}{\sigma}-\frac{\left|t-t^{\prime}\right|}{\tau}},
$$

where $\sigma$ and $\tau$ are the (constant) spatial and temporal decorrelation scales of the field. Note, to compute the mapping error, we need only the measurement times and locations, and not the measurements themselves.

We define two dimensionless parameters for use in the mapping error analysis:

$$
S_{\beta}=\frac{\beta}{v_{0}} \quad \text { and } \quad S_{p}=v_{0} \frac{\tau}{\sigma}
$$

where $S_{\beta}$ is the non-dimensional flow speed and $S_{p}$ is the nondimensional nominal speed of a vehicle. The vehicle is spatially constrained if $S_{p}<1$ and temporally constrained if $S_{p}>1$ [12]. The length of the sensor swath of a spatially constrained vehicle depends on the spatial decorrelation scale $\sigma$, whereas the swath length for a temporally constrained vehicle depends on the temporal decorrelation scale $\tau$. In this context, a swath denotes the area along the track of the vehicle that exhibits a significant reduction in mapping error due to the vehicle trajectory.
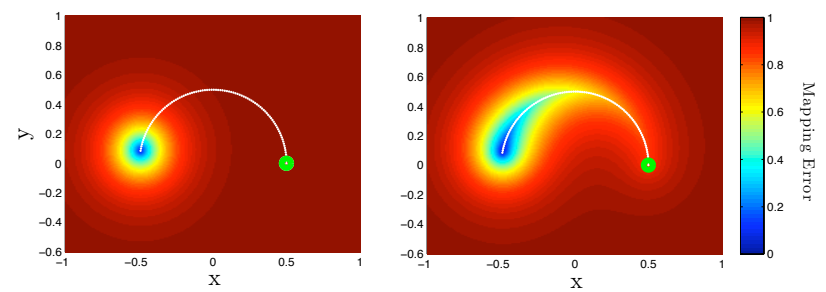

Fig. 2: Comparison of error maps generated for $S_{p}<1$ (left) and $S_{p}>1$ (right). The vehicles travel counter-clockwise from the green dot.

Figure 2 compares the error map of a temporally constrained vehicle with that of a spatially constrained one. Each vehicle travels at the same nominal speed in a circular arc, but the spatial and temporal decorrelation scales are varied such that $S_{p}=.67<1$ on the left and $S_{p}=$ $10>1$ on the right. The vehicle initial position is a green dot, and the trajectories are white lines. The error map of a spatially constrained vehicle appears as an exponential distribution centered at the current location. The error map of a temporally constrained vehicle is characterized by an elongated swath behind the vehicle.

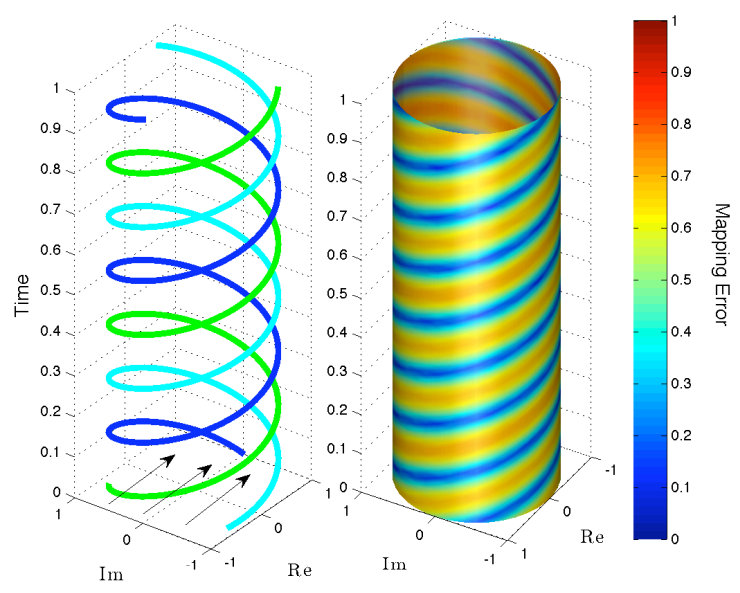

Fig. 3: Three vehicles traveling in circular trajectories shown in the spacetime volume. The error map is represented on the cylinder that encompasses the vehicle trajectories. The black arrows show the flow direction.

Of interest is the error along the track of a periodic trajectory. In the space-time volume shown in Figure 3, a circular trajectory creates a helical pattern. The along-track mapping error is represented as a colormap on the cylindrical surface that encompasses the vehicle trajectories. The colormap can be mapped from the cylinder to a two dimensional space-time 
domain with periodic boundary conditions on the left and right, as shown in Figures 4 and 5. In the 2D representation, the vehicle exits on the right and reappears on the left as it counterclockwise travels around the circle.

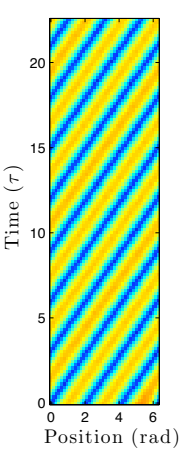

(a) $S_{\beta}=0$

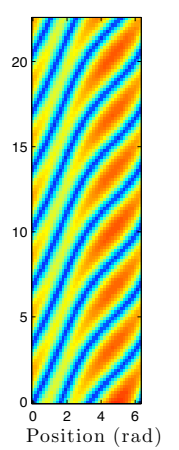

(b) $S_{\beta}=.4$

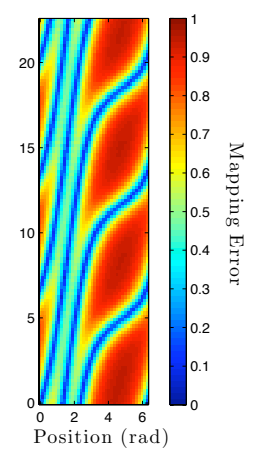

(c) $S_{\beta}=.8$
Fig. 4: Error maps for three values of flowspeed $S_{\beta}$ for vehicles that are nominally spatially constrained with $S_{p}=.7<1$. The timel axis is normalized by the temporal decorrelation scale.

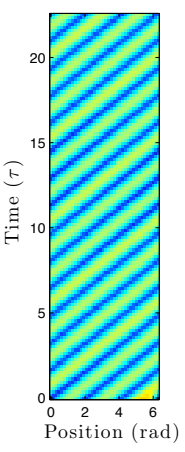

(a) $S_{\beta}=0$

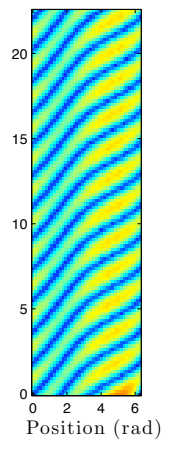

(b) $S_{\beta}=.4$

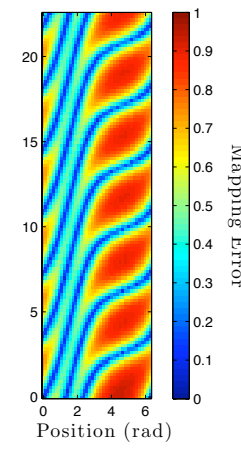

(c) $S_{\beta}=.8$
Fig. 5: Error maps for three values of flowspeed $S_{\beta}$ for vehicles that are nominally temporally constrained with $S_{p}=1.3>1$.

Error maps were created for spatially and temporally constrained vehicles at various flow speeds. Figures 4(a) and 5(a) show the along-track error for the zero-flow case; as the vehicle speed is increased the average error, given by (3) decreases from 0.36 to 0.28 . However, as demonstrated by Figures $4(\mathrm{~b}, \mathrm{c})$ and $5(\mathrm{~b}, \mathrm{c})$ it is not immediately clear what happens to the average error as the non-dimensional flowspeed is increased. We derive a speed controller that minimizes the average error in Section IV-A.

\section{B. Inhomogeneous Field}

We investigate the case where there is a region of the flow that has heightened spatial variability. This case is modeled by the following covariance function [16]

$$
C\left(\mathbf{x}, \mathbf{x}^{\prime}\right)=\sigma_{1}(x) \sigma_{1}\left(x^{\prime}\right) e^{\frac{\left|x-x^{\prime}\right|}{\sigma}-\frac{\left|t-t^{\prime}\right|}{\tau}}
$$

where $0 \leq \sigma_{1}<1$ is the position-dependent correlation coefficient. The correlation coefficient modifies the amplitude of the covariance function but not the decay rate, . The maximum correlation of two locations is $\sigma_{1}(x) \sigma_{1}\left(x^{\prime}\right) \leq 1$. The covariance function (12) reduces the length of the sensor footprint for a spatially constrained vehicle on a circular trajectory.

In this paper, we use a von Mises distribution for the correlation coefficient $\sigma_{1}(x)$ around the perimeter of a circular trajectory:

$$
\sigma_{1}(x)=1-a e^{c \cos (x-\mu)-c},
$$

where the shaping constants $a$ and $c$ determine the height and width of the dip in the correlation coefficient and $\mu$ determines its position. The inhomogeneity representd by (13) can be used to represent a multitude of physical scenarios, such as an area of a salinity field with large gradients [1]. Figure 6 compares the spatial dependence of the covariance function of a homogeneous field and inhomogeneous field. The peak covariance for the inhomogeneous field is less than for the homogeneous field, and its footprint is narrower. The sensor coverage is illustrated in Figure 6 by the width of the of the covariance function at the decorrelation threshold $1 / e$ (shown as a green line).

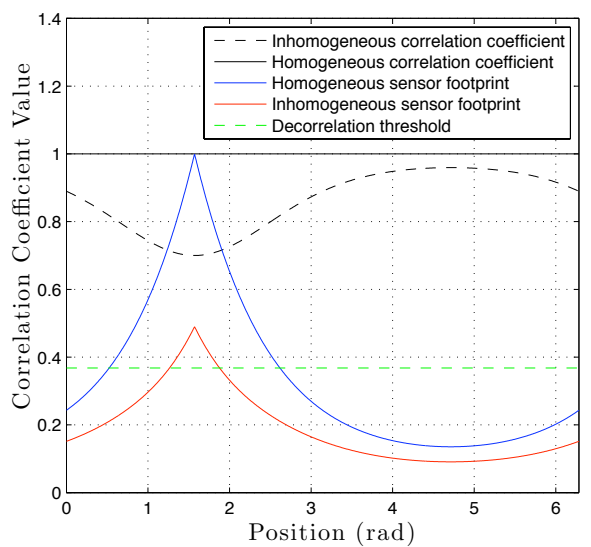

Fig. 6: Correlation coefficient and corresponding covariance function. The blue (resp. red) line represents the spatial dependence of the covariance function for a homogeneous (resp. inhomogeneous) field.

The effect of the spatially variable correlation coefficient on the mapping error is shown in Figure 7(a). The location of the dip is $\mu=\pi / 2$ and the shaping constants are $c=1$ and $a=0.3$. Figure 7(a) shows how the swath length changes as the vehicle traverses the circle, creating a coverage gap where the dip is located and the error is higher. There are also areas where the coverage overlaps; sampling at these locations is redundant. We use this analysis to derive an open-loop speed controller to minimize the average and/or maximum error in an inhomogeneous field in Section IV-B.

\section{Minimization of MAPPing ERror}

\section{A. Homogeneous Field}

In this section, we vary $S_{p}, S_{\beta}$ and $N$ to determine the combination of parameters that minimizes the average error given by (3) in a time-splay circular formation. Figure 8 shows that the trend exhibited in the no-flow cases in Figures 4(a) and 5(a) continue when the flow is non-zero. For a given 


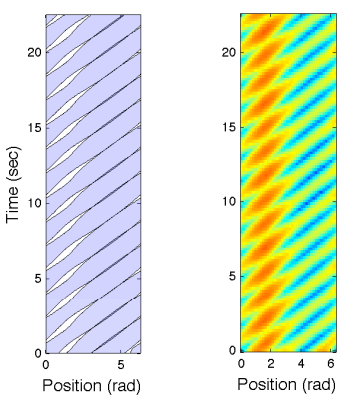

(a) $S_{\beta}=0$
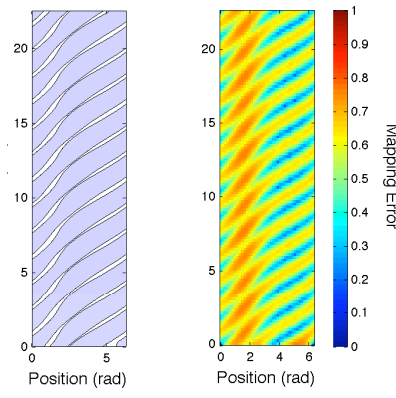

(b) $S_{\beta}=0.3$
Fig. 7: Error map for three vehicles traveling at constant speed around a circle with correlation coefficient given by (13). The dip is located at $\mu=\pi / 2$ and the shaping constants are $c=1$ and $a=0.3$.

flow speed, if the vehicle speed is increased, then the average error decreases. For a given vehicle speed, as the flow speed increases, the average error increases. We also observe that adding more vehicles decreases the average error.

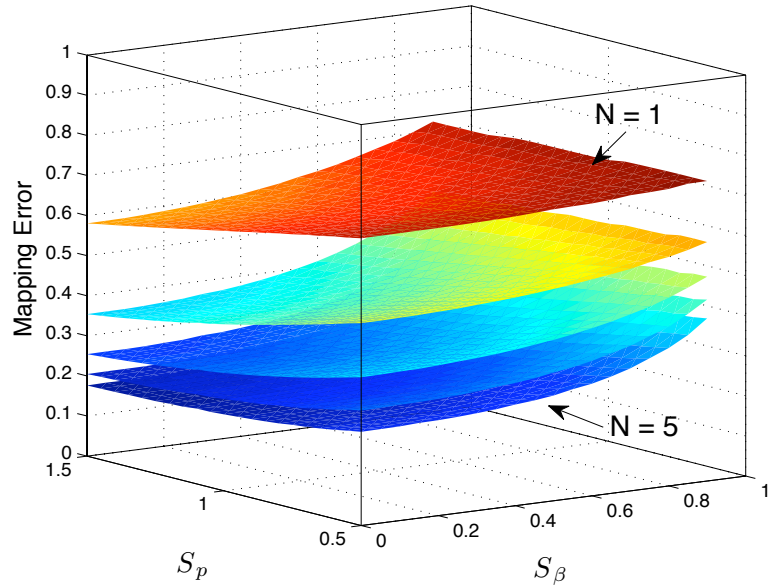

Fig. 8: Average error for up to five vehicles with varying $S_{p}$ and $S_{\beta}$.

From the above observations, we conclude that it is beneficial to have as many vehicles as possible traveling as fast as possible in the absence of flow. This results in the vehicles traveling at constant speed with even spacing. In the presence of a uniform flow $\beta$, this can be accomplished by using the time-splay control law [8] along with a speedcontrol law that drives the speed of each vehicle to a desired value, $s_{0}$. In this section we design the speed controller, $\xi_{k}$, that achieves the desired speed.

Theorem 1: Choosing the controller

$$
\xi_{k}=K\left(s_{0}-s_{k}\right), \quad K>0,
$$

in the model (7) forces the solution to converge to $s_{k}=$ $s_{0} \forall k$.

Proof: Choosing

$$
V=\frac{1}{2} \sum_{j=1}^{N}\left(s_{0}-s_{j}\right)^{2} \geq 0,
$$

yields $\dot{V} \leq 0$, which implies the system will converge to $s_{j}=s_{0} \forall j$.

Note that (14) forces convergence of $s_{k}$ to $s_{0}$ independently of $u_{k}$, which implies that (14) can be used in conjunction with a time-splay control [8] to stabilize a circular formation with evenly spaced particles. For a nominal $S_{p}=$ $s_{0} \tau / \sigma$, the speed control will effectively cancel the alongtrack flow component, which implies the mapping error converges to the values shown for $S_{\beta}=0$ in Figure 8 .

\section{B. Inhomogeneous Field}

In this section, we introduce an artificial flowfield to lower the average and/or maximum error in an inhomogeneous flowfield. This is accomplished by setting the vehicle speed to $s_{k}=\left|v_{k} e^{i \theta_{k}}+\beta\right|$, with flow speed $S_{\beta}=\beta / v_{0}$. An example of how a flowfield can lower the maximum error is shown in Figure 7. In Figure 7(a) the no-flow average error is 0.54 and the maximum error is 0.78. In Figure 7(b) a flowfield with strength $S_{\beta}=0.3$ increases the average error to 0.56 , but also lowers the maximum error to 0.75. In a homogeneous field, the direction of the flowfield, $\arg (\beta)=0$, does not change the error metrics. However, in an inhomogeneous field the relative angle, $\arg (\beta)=\mu$, between the dip location and the flow can also be varied to minimize the maximum and/or average error.

Figure 9 shows how the maximum and average errors in (3) and (4), respectively, vary as the flow direction and speed are changed for three vehicles with nominal $S_{p}=$ 0.7 . Observe that there are flow speeds and directions for which the maximum and average errors are minimized. For example, the maximum error is minimized with a flow direction that is rotated $-\pi / 2$ from the dip angle. Intuitively, the vehicle speed decreases in the dip of the correlation coefficient, which increases the number of measurements in that area and lowers the maximum error. In contrast, the average error decreases when the vehicle speed increases in the dip. While this increases the maximum error, it also increases the number of measurements away from the dip, which lowers the average error.

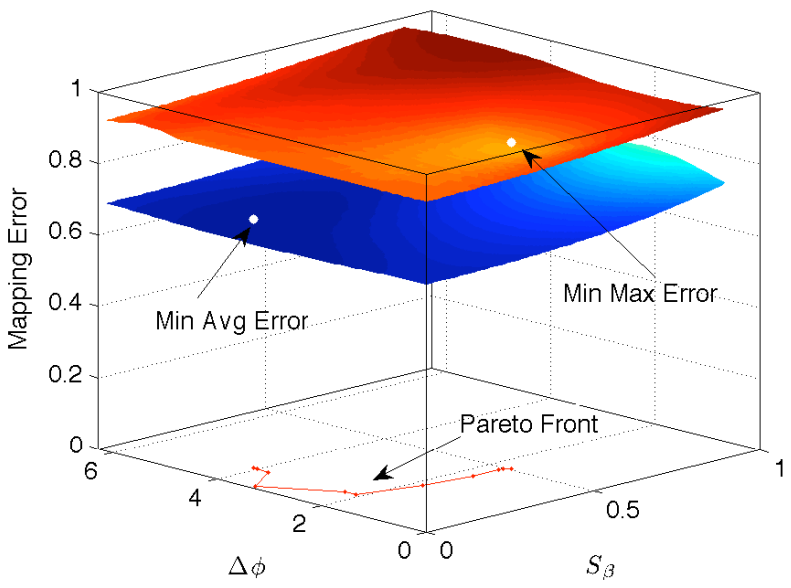

Fig. 9: Maximum and average errors for varying flow speed and direction for three vehicles with $S_{p}=0.7$. 
We now describe the results of minimizing a convex combination of the two error metrics. The combined metric is a weighted sum of the two metrics, $\mathcal{E}=(1-k) \mathcal{E}_{\mathrm{av}}+k \mathcal{E}_{\text {max }}$, where $0 \leq k \leq 1$. The red line in Figure 9 shows the optimal pair $S_{\beta}$ and $\mu$ as the relative weight $k$ varies, i.e., the Pareto front [17]. Figure 10 shows the trajectories that minimize the average and maximum errors - the extreme points of the Pareto front.
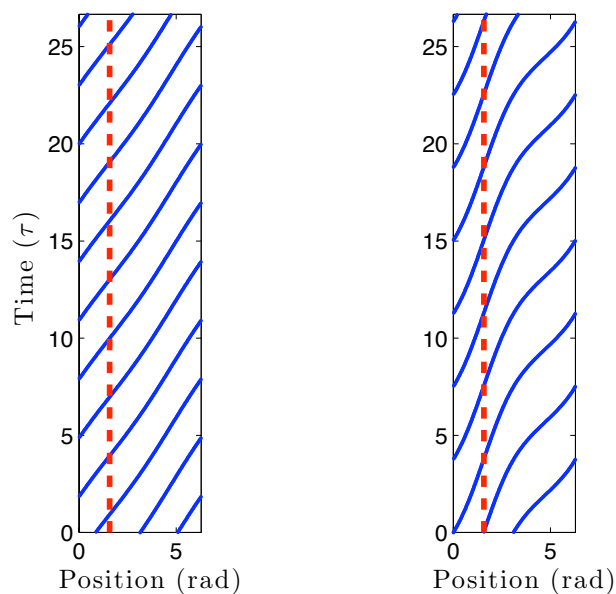

Fig. 10: Optimal trajectories for minimization of average mapping error (left) and maximum mapping error (right) in an inhomogeneous field with $\mu=\pi / 2$ and $N=3$

\section{CONCLUSION}

In this paper mapping accuracy is analyzed for vehicles traveling in homogeneous and inhomogeneous spatiotemporal fields. The mapping accuracy is quantified using average and maximum error as performance metrics. The metrics are evaluated for vehicles traveling in a uniform flowfield. For a homogeneous field, a closed-loop speed controller is provided to minimize the average error. Inhomogeneity is introduced in the spatiotemporal field by varying the correlation coefficient over the vehicle path. An artificial flowfield is used as an open-loop controller to minimize the average and/or maximum errors. In ongoing work we are extending the results in this paper to higher spatial dimensions, in which geometric control of sensor positions may be applicable.

\section{REFERENCES}

[1] Jack Elston, Maciej Stachura, Eric W. Frew, and Ute C. Herzfeld. Toward model free atmospheric sensing by arial robot networks in strong wind fields. Proc. of the Int. Conf. on Robotics and Automation, pages 30903095, May 2009.

[2] S. L. Smith, M. Schwager, and D. Rus. Persistent monitoring of changing environments using a robot with limited range sensing. In Proc. of IEEE Conf. on Robotics and Automation, pages 5448-5455, May 2011.
[3] Russ E. Davis, Naomi E. Leonard, and David M. Fratantoni. Routing strategies for underwater gliders. Deep-Sea Research II, 56:173-187, 2009.

[4] Michael A. Demetriou. Guidance of mobile actuatorplus-sensor networks for improved control and estimation of distributed parameter systems. IEEE Trans. on Automatic Control, 55(7):1570-1584, 2010.

[5] Dariusz Ucinski and Michael A. Demetriou. An approach to the optimal scanning measurement problem using optimum experimental design. In Proc. of the American Control Conf., volume 2, pages 1616-1621, June 2004.

[6] Zhen Song, YangQuan Chen, Jinsong Liang, and Dariusz Ucinski. Optimal mobile sensor motion planning under nonholonomic constraints for parameter estimation of distributed systems. In Proc. of IEEE Int. Conf. on Intelligent Robots and Systems, pages 3163-3168, Edmonton, Alberta, Canada, August 2005.

[7] J Cortez, S Martinez, and F Bullo. Coverage control for mobile sensing networks. IEEE Trans. on Robotics and Automation, 20(2):243-255, 2004.

[8] D. A. Paley and C. Peterson. Stabilization of collective motion in a time-invariant flowfield. J. of Guidance, Control and Dynamics, 32(3):771-779, 2009.

[9] A. Eliassen and J. S. Sawuer. Upper air network requirements for numerical weather prediction. Tech. Note. World Meteorological Org., 29, 1954.

[10] L. S. Gandin. Gidrometerologicheskoe Izdatelstvo Objective Analysis of Meteorological Fields. Israel Program for Scientific Translations, 1963.

[11] P. R. Liebelt. An Introduction to Optimal Estimation. Addison-Wesley, 1976.

[12] D. A. Paley. Cooperative Control of Collective Motion for Ocean Sampling with Autonomous Vehicles. PhD thesis, Princeton University, September 2007.

[13] F. P. Bretherton, R. E. Davis, and C. B. Faundry. A technique for objective analysis and design of oceanographic experiments applied to mode-73. Deep-Sea Res., 23(7):559-582, 1976.

[14] E. W. Justh and P.S. Krishnaprasad. Equilibria and steering laws for planar formations. System Control Letters, 52(1):25-38, 2004.

[15] N. E. Leonard, D. A. Paley, R. Sepulchre, D. M. Frantantoni, and R. E. Davis. Collective motion, sensor networks and ocean sampling. Proc. of the IEEE, 95(1):48-74, 2007.

[16] Andrew Bennett. Inverse Modeling of the Ocean and Atmosphere. Cambridge University Press, 2005.

[17] G. Agrawal, K. Lewis, K. Chugh, C-J Huang, S. Parashar, and C. Bloemaum. Intuitive visualization of pareto frontier for multi-objective optimization in n-dimensional performance space. In Proc. of 10th AIAA/ISSMO Multidisciplinary Analysis and Optimization Conference, Albany, New York, August 2004. 